\title{
On Digital Diplomacy
}

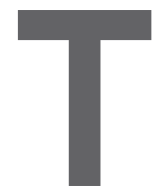

HE TERM DIGITAL DIPLOMACY might have two interpretations. One is the conduct of diplomacy through digital means. The other is diplomacy concerning digital technologies. Both interpretations are addressed in this column. Interestingly, the term protocol in the computer communication sense was adopted from its use in diplomatic terms. Protocols are practices, procedures, and forms in the diplomatic world. In the world of computing, they are procedures and formats for information exchange. The conduct of diplomacy through digital means is, at first glance, a natural extension of face-toface (F2F) and written diplomacy. From 1845 to 1865 , telegraphy was a terrestrial service and quickly put to use in commerce, diplomacy, and war. ${ }^{\mathrm{b}}$ When the first, lasting telegraphic trans-Atlantic cable was laid in $1866,{ }^{\mathrm{c}}$ telegraphy became an important part of rapid diplomacy on an intercontinental scale. The invention of the telephone provided for real-time interactions, the value of which became especially apparent in the wake of the October 1962 "Cuban Missile Crisis" leading to the installation of a "hotline" between the White House and the Kremlin. Facsimile transmission capability arrived in quantity in the 1980s, transforming diplomacy once again.

With the arrival of the Internet, electronic messaging has become widespread and in informal use worldwide. Formal diplomatic communications

a O.S. Adesina, J. Summers (Reviewing Ed.) Foreign policy in an era of digital diplomacy. Cogent Social Sciences 3, 1 (2017). DOI: 10.1080/ 23311886.2017.1297175

b President Abraham Lincoln famously and avidly read dispatches from the war front during the Civil War, for example.

c Earlier efforts beginning in 1854 did not succeed or failed quickly. are still largely conducted by fax, post (including overnight delivery), and telephone as well as venerable F2F communication. Electronic mail is typically used for internal but not external communication owing to the potential for misrepresenting the origin of public email messages ("spoofing"). Even that is slowly changing as various forms of strong authentication become available.

The arrival of the World Wide Web (WWW) in 1991 heralded a new era in information production and sharing. From the diplomatic perspective, it offered an open source for intelligence about events in the world and also an avenue to engage in what some call soft diplomacy by which is meant pursuit of diplomatic aims by means of suasion and public pressure. Concurrent with the evolution of the WWW, cloud computing has emerged as the natural successor to the time-sharing systems of the 1960s. Cloud computing and smartphones have contributed to two more recent dramatic changes in the online environment that are upending traditional diplomatic communication. The first is the arrival of social media, most notably Facebook, Twitter, Instagram, Tik-Tok, WeChat, and YouTube, among others. The second is public and private videoconferencing. Videoconferencing is not new. Indeed, its origins lie in the 1960s and experiments with this medium were conducted on the American ARPANET in the 1970s. The COVID-19 pandemic, however, has launched videoconferencing over the Internet into orbit. To account for lockdowns and social distancing, videoconferencing systems like Zoom, Teams, Meet, among others, are now in daily use worldwide. We are locked in front of our laptop screens, not only by email, chat, and surfing the Web, but by videoconferences and webinars going day and night.
On the negative side of the ledger, social media have become avenues for the spread of misinformation and disinformation and have been used to foment civil unrest. The latter is often triggered by the distribution of false information and reinforced by the use of botnets ${ }^{\mathrm{d}}$ and fake accounts in social media. Indeed, such abuses highlight the growing recognition in government and diplomatic circles that abuse of the Internet is a national and international problem that needs attention (See S. Cresci's article on p. 72). Hence the second interpretation of digital diplomacy: diplomatic negotiations about dealing with the abuse of digital infrastructure. The canvas for this debate is enormous. There are billions of digital devices in use today from mobile smartphones, laptops, desktops, tablets, and a growing body of devices called collectively the Internet of Things. Hacking of these devices along with the distribution of malware (that is, harmful software) constitute major hazards in the online world. These hazards are made all the more difficult to deal with because the Internet is global, and perpetrators may be in one jurisdiction while the victim is in another.

It is apparent that the world needs thoughtful and technically credible debate on alternatives for containing the problem of harmful behavior on the Internet. Perpetrators must be identified, and international norms and agreements established for bringing them to justice. This is a task for diplomacy about digital technology and its use and abuse, and computer scientists have a serious role to play in this discussion. c

d A "bot" is a computer that has been hacked to become part of a large network of machines that can be used to mount denial of service attacks, spread spam and misinformation and reinforce tweet storms, among other things. A "botnet" is a collection of bots.

Vinton G. Cerf is vice president and Chief Internet Evangelist at Google. He served as ACM president from 2012-2014.

Copyright held by author 